\title{
Environmental Management Systems and Sustainability in SMEs
}

\author{
Satya Shah ${ }^{1, a}$, Elmira Ganji ${ }^{1}$ and Syed Hasan $^{2}$ \\ ${ }^{1}$ Faculty of Engineering and Science, University of Greenwich, UK \\ ${ }^{2}$ Industrial and Manufacturing Engineering, NED University of Technology, Pakistan
}

\begin{abstract}
Environmental sustainability in manufacturing sector has been allocated a major consideration in the international literature. Due to growing concerns over the high effect of SMEs on world manufacturing industries and their contribution to pollution; this research attempts to focus on the key parameters that interact in the application of environmental management system, taking into account the main features of SMEs and also the integral role of industrial entrepreneurs in inspiring their firms' approaches. The paper explores the potential opportunities which enable these enterprises to move towards organizations with high level of responsibility regarding environmental protection in order to provide a healthier life for future generations. Case investigation is carried out on an adhesive manufacturing company, which covers a notable market share within the sector. The research identifies that the company requires developing both internal and external entities within an explicit plan to revolutionize the recruitment patterns. Given the lack of adequate studies in adhesive technology, more researches are recommended in the future to consider the sustainable innovations on a broader sample of adhesive manufacturing companies to perform the life-cycle analysis due to the harmful organic compounds and toxic vapours of the adhesive products.
\end{abstract}

\section{Introduction}

In the last two decades, the concept of sustainability and accountability has become an emerging characteristic of the enterprises worldwide. Improving significant aspects of a company success such as economic, social and environmental performance; sustainability defines a strategy to transform businesses towards long-term prosperity. The Brundtland Commission (WCED) defined sustainable development in 1987, as "seeking to meet the needs and aspirations of the present, without compromising the ability to meet those of the future" which became a classic and common definition [1]. Nowadays widespread idea of the triple $\mathrm{P}$ of business (Profit, People and Planet) which is delivered by [2], describes the term sustainability appropriately. Therefore, environmental sustainability would be a significant factor of every decision-making, from purchasing raw materials to products and services that firms provide. Going green is at the heart of the sustainability and involves some guiding principles to provide the deepest view of how the business can have impacts on our planet and build a better life for future generations [3]. In other words, green strategies focus on propelling just one of the sustainability goals which is environmental stewardship [4].

ISO is originally a Greek word meaning "Equal" and it was initially an organization for product development and safety standards which altered its traditional way by evolving a series of quality management standards [5].

\footnotetext{
a Corresponding author: s.shah@gre.ac.uk
}

Implementation of the EMS and adopting a certification such as ISO 14001 or EMAS act as sustainable evaluation tools to provide visual assessment, monitor the production waste and environmental performance. A comprehensive definition for EMS is given by ISO.org, as "organizational structure, responsibilities, practices, procedures, processes and resources for determining and implementing environmental policy". Thus, EMS would be the effective and positive bedrock for companies' continual improvements that also assist them on their path towards sustainability [6].

\section{Literature Review}

The review of literature seeks to outline the conceptual framework of the research by presenting a review of advantages, disadvantages, motivations, drivers and hurdles towards Environmental Management Systems (EMS) in order to prove the necessity of the environmental sustainability practices within the SMEs. In particular, adhesive technology characteristics considering major problems correlated with optimizing sustainability and its environmental impacts will be examined.

\subsection{Importance of SME on Business Factors}

Since the priority of any firm is its survivability and better performance, SMEs are not only an exception, but also have a significant impact in most of the market economies. Research studies indicated that SMEs make 
up about $90 \%$ of all global enterprises and many of them are suppliers over the supply chain [7]. SMEs share of global environmental pollution is $70 \%$, with the majority of manufacturing sector [8]. Moreover, $0.4 \%$ of all the SMEs belong to the enterprises with more than 100 employees. Climate change is seen as the greatest environmental challenge facing the world today and is considered as the key focus of international, European and UK policy. With climate change considered as an important factor within any environmental and governmental policies, the study aims towards focusing on the importance of Environmental and Climate change aspects through sustainable development and Environmental Management systems within SMEs.

The existing literature on the business response to climate change seems to be largely normative rather than empirical in nature and tends to focus towards larger firms rather than small and medium enterprises (SMEs). Similarly, government policies on climate change often focus predominantly on larger firms, with less emphasis on the roles that SMEs can play. Yet, as SMEs constitute $99 \%$ of UK businesses, provide $43 \%$ of private-sector employment and may account for $20 \%$ of total UK carbon emissions understanding their environmental impact and engagement, both generally and with respect to climate change, seems highly important [9].

Identifying a firm internal and external changes and profitability benefits through adoption of sustainable strategies are fundamental factors in healthy dynamic businesses. Hence, there is a growing concern to promote SMEs to make investments in sustainable business practices, particularly in developing countries as it has been noted that the industrial activities carried out within the SMEs contributes towards environmental pollution. This would be also as a result of poor financial resources, lack of regulations and inadequate expertise to deal with complex technologies. Utilising economical and simple solutions facilitate a foundation for developing countries to implement effective systems for environmental protection without the need to spend long time periods or having high scientific backgrounds, high costs and high level technologies. Scrutinizing SMEs characteristics to face green or environmental management issues and sustainability criteria, helps researchers recommend applicable mechanisms for such. On the other side, SMEs may confront many pressures on their management strategies to the way of being environmentally friendly. Inadequate financial resources, lack of technical expertise, low level of research and development, management weaknesses and their visions that SMEs have little environmental impact are the major barriers for SMEs social responsibility and competitive advantage [10]. Therefore, the strategic choices of entrepreneurs and managers play a vital role in organizational growth and sustainable development of the firm in the long-term perspective. This research seeks to address the gaps in literature regarding the actual effectiveness of considering environmental sustainability practices with a particular attention to the traditional management methods, lack of understanding of environmental risks and lack of proper information availability within SMEs, specifically in manufacturing sector. The author also tends to look at the recruitment practices in SMEs as a part of the research.

\subsection{EMS Objectives for SMEs Performance}

Numerous benefits of adopting EMS by SMEs have been categorized by [8] into two main groups: Internal Benefits and External Benefits. Internal benefits include many organizational improvements, financial savings and payback periods of investments within the company. Moreover, EMS implementation provides new interactions between personnel and management and results in intangible advantages such as employee morale and social responsibility (MSR) enhancement, communication and skills improvements, increasing knowledge and altering staff's attitude which are very important for SMEs performance. In terms of External benefits, companies find more competitive and business rewards. Therefore, they attract new business partners and increase satisfaction of customer requirements. In addition to the commercial benefits, companies find positive outcomes such as environmental achievements, assured legal compliance, energy consumption minimization and waste reduction. As a result, relationships with stakeholders, company image and reputation will be improved.

The main objectives of EMS which move companies towards systematic approaches development has been defined by [6]. Applying the concept of EMS not only assists SMEs to reduce energy consumption, pollution and hazardous wastes in the chemical industry; but also lead them into conservation of resources, increasing productivity and reducing costs. It develops awareness of environmental issues and responsibilities among entrepreneurs. Furthermore, SMEs will be encouraged to apply an environmental management system in their production processes. Research studies introduced EIS which help SMEs to review comprehensive environmental information relevant to the company operations and facilities. EIS can be used as an effective tool to measure, benchmark and monitor the environmental performance in a time sequence analysis. The environmental indicators enable company managers to detect the market prospect, cost reduction potentials, compare their firm with industrial standards and importantly, to better decision-making [6]. However, ISO 14001 does not require indicators development but they could be obtained through ISO 14001 standard. The studies pointed out that eco-mapping is a visual assessment tool particularly designed for SMEs to reduce their environmental documentation quantities. Using this tool includes maps for various entities of the company, such as water map, energy map, and material and resource flow map [6]. Furthermore, flowcharts such as process maps provide a general and instant overview of company operations, starting with material inputs ending up with waste disposal and distribution [11]. Both these evaluation tools assist and prepare SMEs on their journey to environmental management system and sustainability. 
Quantitative evaluation of environmental management system is required to help us determine the effectiveness and value of such a system. Assisting particular sector if SMEs, helps to focus on homogenous tools and approaches without necessity of high cost modification or application. Moreover, using visual assessment and evaluation tools already exist and necessitate the contribution and commitment of all personnel which is a key success towards EMS implementation. These tools help manufacturing sector appropriately but they still remain heterogeneous and need to be integrated. In general, three main factors answer the "Why" it is necessary to adopt EMS by SMEs. Firstly, implementing EMS brings high efficiency and cost savings. Moreover, many bank insurance companies give predominance to organizations with low environmental risk. Secondly, market demand for products and services with better environmental preferences such as "green consumerism" would be another driver. Thirdly, effective EMS brings firms fewer risks of breaking the laws and also fewer environmental incidents [6].

\subsection{SMEs Motivations towards Environmental Approaches}

Different Scenarios exist for SMEs to optimize sustainability and create competitive advantages and were discussed by [7]. These motivations enable SMEs to develop into valuable sustainable investment target points for larger firms; therefore, they become sustainable suppliers in both global and local supply chain. If SMEs work collaboratively and create networked entities in sustainable marketplace, which is nowadays facilitated by globalization of communication technology, it can offer them the opportunities for better economic performance where the MNEs (Multinational Enterprises) are less successful.

Considering SMEs from an upstream view as buyers from suppliers, more pressures can be exert by them in order to take into account the three dimensions of sustainability in order to provide a sustainable supply chain [12]. Furthermore, other research studies also claim that motivations inspiring the sustainability concept for the SMEs are in a wide range, including internal organization improvements, legal requirements, competitive advantage, reputation concerns and increasing profitability [13]. Three fundamental phases and motivations have been identified for corporate sustainability by [14]. In this regard, sanitizing as compliance driven health or safety efforts, controlling as eco-efficiency driven environmental management and integration of all the components of the company into business decisions as a value creator have been mentioned.

Exploring the strong drivers such as customers, legislation and social responsibility of SMEs environmental processes like disposal, recycling and EMS, it is necessary to address their impact on company performance. Achieving this goal, a sample of 500 Turkish SMEs was investigated by [15] using a crosssectional design. They considered regulation as a low- level motivator towards environmental pollution in Turkish SMEs. They pointed out that governments can also act with other beneficial techniques such as building waste treatment facilities in industrial areas and encourage the SMEs to develop their environmental projects. The important point is that besides environmental issues, government policy should also result in SMEs growth. Moreover, OEM as an element in supply chain could offer some help to their suppliers in order to provide green design, processes or certifications. In the factor analysis, the researchers have eliminated some of the drivers triggering environmental processes due to the poor loadings on their intended factor. Removing short-term profits allows long-term benefits to be more investigated along with market share, firm image and competitive advantage [15]. One significant problem which has not been solved yet is that SMEs are in an ambiguous situation and that the confusion among different entities controlling the environmental programs always exists, and some SMEs operate without accurate inspection. Moreover, a huge investment is needed to implement recycling processes or EMS.

Assessing the motivation of five German SMEs to implement and certify EMS was part of another research studies [16]. Among the small and medium-sized energy and gas companies in Germany, organizing companies to deal with environmental regulations and domestic competition, finding cost cutting opportunities and increasing efficiency for competitiveness enhancement are frequently mentioned as strong motivators to adopt EMS. These companies reported air and waste emissions reductions, energy and water conservation, safety and incidence reduction as positive consequences of ISO 14001. However, it is essential to perform more researches to explore whether the benefits and positive impacts of certifying environmental management system can accomplish the strong motivations of doing so. Furthermore, among all the benefits of SMEs, cost savings documentation, increased competitive advantages and operational improvements are more difficult to bring into focus.

\subsection{Environmental and Sustainability Practices and Strategies}

Key parameters and strategies which interact with implementation of environmental management tools have been described and analysed by taking into account the specific characteristics of the SMEs [10]. According to Figure1, in the First stage of the strategy it is noted that environmental tools adoption does not require extra financial support or access to capital [8] [10]; as SMEs may have higher risks, information limitations and higher costs for implementing EMS. Since the small firms usually consist of an operative unit which is being run by a single individual owner-manager having the absolute authority, the Second stage is to identify that the mind-set of the manager in risking, embracing change, innovation, growth and social responsibility exert a high influence on the development of firms and choice of strategies and policies. 


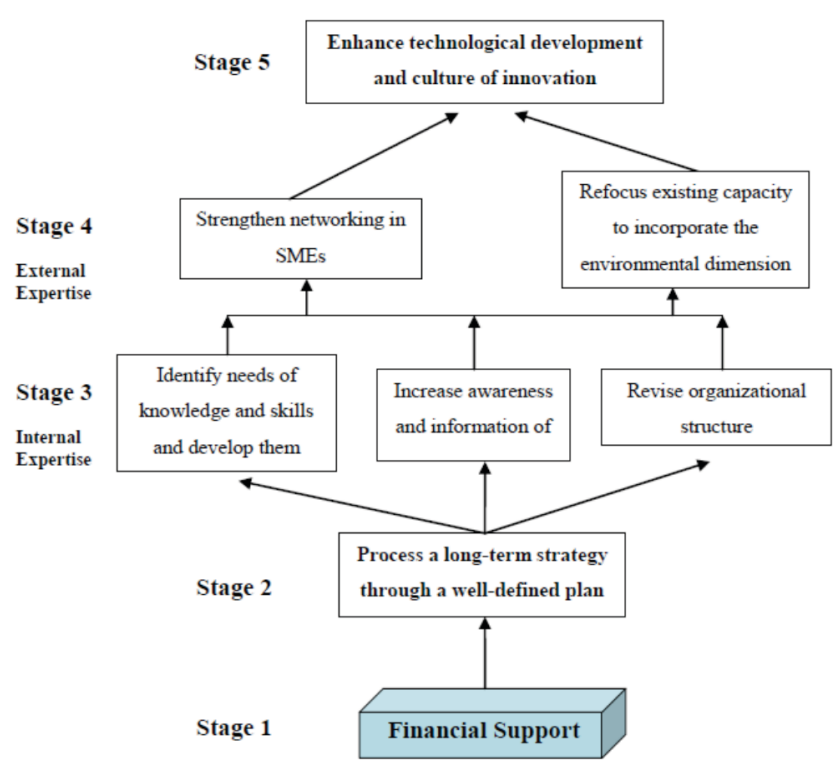

Figure 1. Proposed Strategy for Environmental Activities in SMEs [10].

The Third stage indicates the necessity of internal expertise, higher qualifications of managers, attracting high skill labour and increasing awareness on waste minimization in SMEs. Moreover, SMEs organizational structure need to be revised as a small team who is generally responsible for all the important aspects of the business including key decision makings, dealing with regulations and identifying markets. In doing so, SMEs have to shift responsibilities to qualified workers in order to facilitate collaboration and a democratic workplace which relies on high knowledge and expertise. In the Fourth stage, using the external expertise has been mentioned. Eventually on the Fifth stage, the need for technological development and a culture of innovation is illustrated. In general, in the case of packaging they mentioned that legislation, producer responsibility obligation and increasing pressure on companies related to environmental issues will result in attracting more expertise and advice in order to implement environmental management tools with the new regulations.

Strategy elements that address several important issues for adopting environmental management tools need more research, since they might develop or become invalid in the future. In SMEs, the transit packaging is used to supply materials and finished products that is the treat for the packaging waste. This information could also be utilised for developing an export system. Research investigated development practice and innovation theory within the SMEs which will lead sustainable innovations into practice. In that research it is shown that sustainable innovations mostly rely on technological processes improvements and reduction in production costs [17]. The case of PRIMA project (rubber and plastics industry) was selected, in order to interview with their 26 companies and gather the appropriate data to study the triple $\mathrm{P}$ in business through them. In the specified case the dominant role of the managers is considered as one of the SMEs behavioural advantages to undertake the sustainable innovation activities. Moreover, flexibility of the organization is another benefit for SMEs especially the smaller companies, as a little bureaucracy and personnel motivation will result in more efficiency in innovation projects.

In contrast, managers could have disadvantages regarding to their short-term focus and also weaknesses in managerial skills such as decision making. Plenty of sustainable innovation activities indicate that attaining a determined concept for sustainable innovation seems not to be easy in the short-term. Therefore, further researches should be done to compare the influence of internal and external factors in SMEs to the way of their sustainability corporations. Furthermore, better understanding of the sustainable innovation techniques, companies' stakeholders and staffs could perform tasks more effective to make developments in company performance.

\subsection{Environmental Impacts of Adhesive Industry}

Studies had sought to find out the concept of environmental sustainability within the adhesive industry. Adhesives are mostly consisting of polymers and derived from petroleum feedstock which is non-renewable resources [18]. Moreover, in their manufacturing process, a large-scale energy with pollutions including greenhouse gases used as input and emissions of organic pollutants will be generated in output. In terms of resources, studies also declare that adhesives produced from renewable vegetable and animal resources were being used for a long time. In addition, the pressures towards sustainable practices caused investigations to develop new polymers from renewable biomass, using fermentation techniques or controlled pyrolysis [18]. Adhesive products are a main source of volatile organic compounds which are harmful to the atmosphere as they cause photochemical smog and also lung diseases such as asthma. Furthermore, organic vapours have the same influence as greenhouse gases due to absorption of infra-red radiation [18].

However, several adhesives such as rubber to metal bonding which were produced as organic solvents are based on aqueous emulsions at present. Residues of some of the compounds used in additives and adhesives might be released into the environment and reported for disruption of endocrine system as well. According to European Union "REACH" regulations (Registration, Evaluation, Authorization and Restriction of Chemical); more obligations should be applied to the industries to substitute safer materials by endocrine disruptors.

Taking into account the two important factors including raw material inputs and discharging wastes outputs from and back to the ecosystem, the laws of the Thermodynamics can be considered. In accord with the second law of Thermodynamics, in this case, system begins with highly ordered raw materials with low entropy and moves towards increasing entropy and finally ends up with disordered wastes with maximum entropy which is called the state of "Thermodynamic Equilibrium". Therefore, the petroleum with low entropy 
is burnt to produce carbon dioxide with high entropy. On the other hand, constant rate of growth (size of the economy or resource) relies on the growth rate in mathematical terms. Therefore, it is clear that as long as the economic system pursues endless growth, sustainability could not be achieved by adhesive technology and this fact is well ensured by Thermodynamics laws. However, many researchers have tried to find a solution for environmental impact of particular adhesive products; still it is necessary to consider the total environmental aspects of the engineering framework in which the adhesive technology is being developed. Improving the engineering efficiency towards products sustainability and reducing the environmental impacts of adhesive industry, a complete "life-cycle analysis" would be found.

\subsection{EMS Disadvantages for SMEs}

The disadvantages of EMS have been grouped [8] into three categories. Some studies indicate that cost of certification/validation, cost and quality of advising consultant resulted in SMEs dissatisfaction with implementing the EMS. In this regard, lack of rewards, restriction of linking the EMS to quality systems and complexity of this approach are other factors which need to be taken into account. According to the research, legislation and regulators are more significant for environmental improvements in SMEs than customers and this fact shows the key role of regulations in SMEs environmental strategies [8]. Investigating the adoption of formal EMSs across the European Union, studies identified regarding EMAS and ISO 14001. According to reliable commercial sources, comparing ISO 14001 standard popularity to EMAS, it can be estimated that the percentage of SMEs registered to international guideline ISO 14001 would be higher than for European EMAS. This is also widely reported in the analysed studies. Due to the variety of enterprises in the SME sector, it is not evaluated as a homogenous group of companies.

Therefore, SMEs have a diverse and heterogeneous nature and the studies which probe this sector are too general. The paper conducted also identified the limitation and it is recommended that in the further research, specific sub-groups of SMEs in terms of size and sector diversity be considered [8]. In summary, the literature demonstrated that environmental management system and sustainability strategies have been mostly employed in developed countries such as European countries. Implementing EMS in SMEs, different techniques and strategies were studied in order to gain a perception about the criteria of the environmental sustainable practices. However, there is lack of literature regarding adhesive technology environmental impacts. Objective 1 and 3 was fulfilled through wide-ranging literature studies. In the next chapter, a detailed description of all features of the design and research settings will be presented through main principles for the research approach.

\section{Research Methodology}

This research is based on both qualitative and quantitative data which is facilitated through detailed company survey using site visits, themed interviews and questionnaire administration. Furthermore, reviewing the history and background of the company, all the publicity available materials such as company website and brochures were used within the research. The proposed research seeks to describe the characteristics of a sustainable manufacturing business by pursuing environmental management strategies based on the collected data from document research, industry association data from the interviews and also any government environmental regulations, therefore it would be a descriptive research.

The population of the study is the personnel of Company $\mathrm{X}$ consisting of the shareholders, senior managers, engineers and high-level personnel due to their primary knowledge; current, accurate and factual data they can present for the research purpose. As a result, the researcher will be able to draw assumptions and derive findings based on the existing information and perform the analysis in the higher steps. In Company X, five main parties were involved in meetings and helped the author to write up the observations and findings of the production line and surroundings during two days company visit; comprising HSE department (Health and Safety protection experts), quality control management, planning management, foreign purchasing management, and technical management. Moreover, the five owners (shareholders) and the board of director of the company offered an opportunity to ask some key questions and take their feedback regarding the most significant challenges they face in the company.

\section{Results and Discussions}

Different levels of managers and personnel were engaged in completing the survey questionnaires. The respondent to the survey questionnaire consisted of owners $(17.5 \%)$, board of directors $(3.5 \%)$, top managers $(24 \%)$, engineers $(27.5 \%)$ and other high-level personnel $(27.5 \%)$. In total 29 responds were received for the Question 1, 2, and 3 for the further analysis. Question 4 was only administrated to the company owners, since it was only related to their job responsibilities. The key questions identified within the research study are as shown below:

1. In which department do you work? What is your task within that department?

2. How important are these stakeholders' pressures listed below in influencing your company attitudes towards environmental performance and are known as main drivers for adoption of formal EMS?

3. How important are the factors listed below in motivating your facility to seek for ISO 14001 and sustainability practices?

4. From your perspective, what are the top 5 challenges your firm faces generally? 


\subsection{Influence of Stakeholder's Pressures to adopt EMS}

In accordance with the second question, all the 29 respondents were replied to all parts of the questions, and the respondents indicated that a variety of stakeholders exert strong influences on their environmental performance improvement.

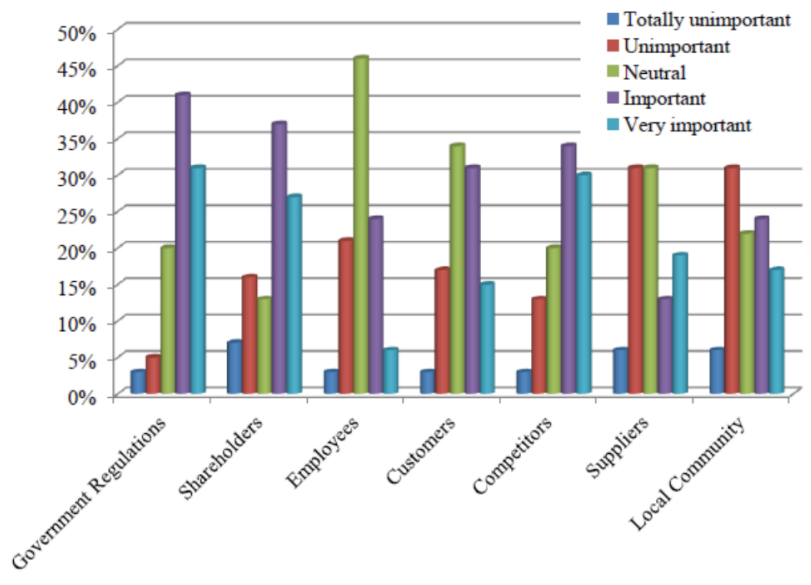

Figure 2. Influence of Stakeholders pressures towards adopting EMS.

As it is evident from figure 2, the three stakeholders commonly mentioned as applying pressure to undertake EMS were competitors, government regulations and shareholders with $31 \%, 30 \%$ and $27 \%$ respectively. The power of inspiration and significant role of these three entities in changing the whole SME approaches towards thinking about environmental concerns and working in a sustainable organization can be realized from the following chart. On the contrary, the local community and suppliers were mainly mentioned as unimportant components with no pressure and little influence on firm environmental attitude by $31 \%$ of each. With respect to the employees, the respondents recorded $46 \%$ neutral answers which can be interpreted that the environmental approach and awareness of the employees is not sufficient and they seem to have no orientation about these issues. This can be as a result of cultural and existing gaps in a developing country, meaning that it is not a long time that the public society got familiar with environmental importance of today's world.

\subsection{Motivation towards adopting EMS}

Figure 3 illustrates the significant business elements and their level of encouragement for the owners, top managers and the rest of the staff of Company which can revolutionize their way of thinking about the wide range of environmental program advantages and build a foundation for them to uptake a sustainable agenda and achieve its great results. Competitive advantage was most frequently rated as a very important motivator, by $31 \%$ of the respondents and only $10 \%$ of them judged it as unimportant. $24 \%$ noted that they can be highly motivated by profitability and cost savings.

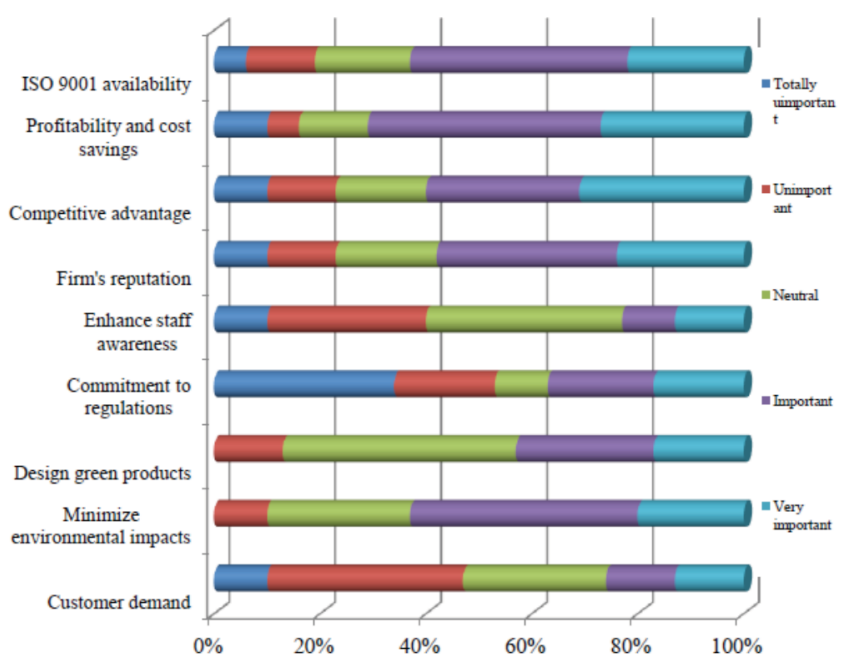

Figure 3. Motivation Factors towards adoption of EMS

By contrast, commitment to the government regulations was judged as totally unimportant by a massive $34 \%$ of the respondents. Considering the respond to customer demand, a large percentage of $37 \%$ mentioned it as an as an unimportant factor which is a surprising statistic showing that the house customers or even industrial customers do not consider the negative impacts on the environment or the green certificates the company might have. This is also mentioned by the planning manager of the Company $\mathrm{X}$ during site visits.

\subsection{Business Owners Challenges}

With regard to the fourth question, the total number of 5 participants including owners (shareholders) of the Company $\mathrm{X}$ participated to the questionnaire. The question has sought to discover the top 5 most challenging aspects that they currently have to tackle in their business.

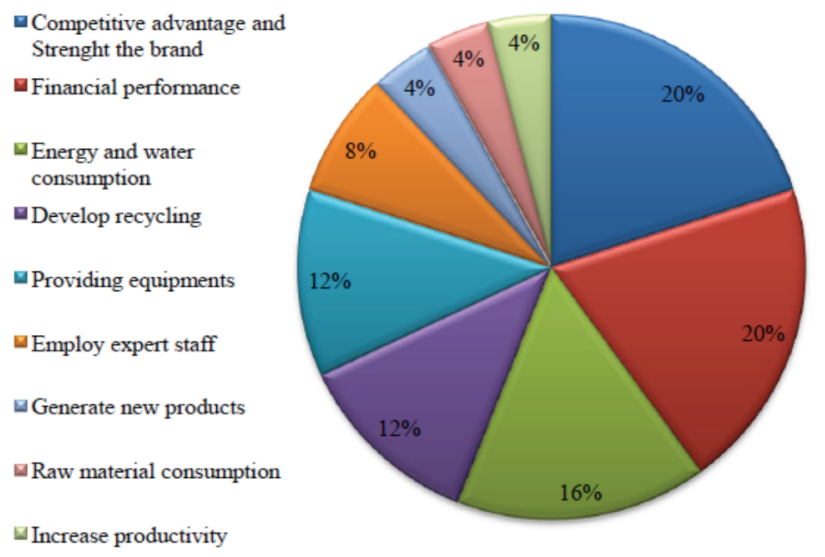

Figure 4. Business Owners Challenges within their Environment

Figure 4 indicates that the top five challenges the managers encounter were strengthen the Razi brand $(20 \%)$, increasing financial performance $(20 \%)$, energy and water consumption (16\%), providing equipment's $(12 \%)$ and recycling development $(12 \%) .8 \%$ of the respondents were also largely regarded to be so important by $24 \%$. Hence, it is evident that employing expert staff, 
generating new products, increasing productivity and reducing raw material consumption have a minor priority and currently their key focus is towards competing with the growing market of SMEs to save their costs. This can be achieved by consultations with professional advisors and also employing high-skilled staff instead of those who are old and have old-fashioned insights into environmental sustainability issues.

\section{Conclusions}

The research was set out to scrutinize the concept of environmental sustainability and has determined the practicality of EMS within an SME in a developing country; explored the incentives and supply chain impressions for the standard system adoption, and the role and impact of management strategies on an organization sustainability and environmental developments. The study also has sought to argue the success of sustainable practices in SMEs which confront with internal and external hurdles of doing so. Main consideration was given to shifting managers' longestablished attitudes, tackling the employees' indifference to environmental issues and also making coordination with the upstream and downstream stakeholders of supply chain. It would not be surprisingly that the findings of the research proved the significant role of owner-managers of SMEs as absolute authorities and decision makers in SMEs who have the power to embrace changes and address environmental risks by developing EMS. Hence, they need to be aware that their company exists not only to generate profit, but also to create a positive revolution in the world by actions to deal with the global issue of sustainability. They need to perceive that a sustainable business is more efficient, more robust and has closer ties with the stakeholders. In essence, based on the findings of the research; it seems that implementing EMS within the investigated SME absolutely has too many advantages that outweigh the disadvantages; such as improving firm images, enhancing environmental responsibility, achieving cost savings, profitability in the long-term and business prosperity.

The authors have attempted to discover appropriate answers concerning initial questions of the study which helps to delineate the scope of the research. The key question was to identify the factors which can motivate SMEs to adopt EMS. Moreover, advantages and disadvantages of environmental practices were compared together in order to find out the highest possible benefits that company can achieve through this journey. However, in the developing countries due to the economic, finance and cultural gaps still there is a long journey to the adaptation with the green management and sustainability practices to the businesses. The barriers of sustainable practices adoption in a developing country were explored, considering the major challenges of business entrepreneurs and the vulnerability of SMEs.

\section{References}

[1] Brundtland Commission, World Commission on Environment and Development (1987). Our Common Future. Oxford: Oxford University Press (1987)

[2] J. Elkington. Cannibals with Forks: The triple bottom line. Oxford Press Publishers: Capstone (1999)

[3] J. Kaplan, J. Greening Your Small Business: How to Improve Your Bottom Line, Grow Your Brand, Satisfy Your Customers-and Save the Planet. New York: Penguin Group, pp. 13 (2009)

[4] F. Roettgers, Going Green Together: How to Align Employees with Green Strategies. Environmental Pioneers (2011)

[5] G. Woodside, P. Aurrichio, P, J. Yturri. ISO 14001 Implementation Manual, McGraw-Hill, pp. 3 (1998)

[6] A. Zorpas. Environmental management systems as sustainable tools in the way of life for the SMEs and VSME, Bioresource Technology, 101(6), pp. 1544-1557 (2010)

[7] S.B. Moore, S.L. Manring. Strategy development in small and medium sized enterprises for sustainability and increased value creation. Journal of Cleaner Production, 17(2), pp. 276-282 (2009)

[8] R. Hillary. Environmental management systems and the smaller enterprise. Journal of cleaner production, 12(6), pp. 561-569 (2004)

[9] H. Williams, A. Schaefer, A. Small and Medium Sized Enterprises and Sustainability: Manager's Values and Engagement with Environmental and Climate Change Issues., Business Strategy and the Environment, Vol 22: pp 173-186 (2013)

[10] D. Perez-Sanchez, J.R. Barton, D. Bower. Implementing environmental management in SMEs. Corporate Social Responsibility and Environmental Management, 10(2), pp. 67-77 (2003)

[11] US EPA. Integrated environmental management systems: implementation guide. Washington DC: U.S. Environmental Protection Agency (2000)

[12] F. Ciliberti, P. Pontrandolfo, B. Scozzi. Investigating corporate social responsibility in supply chains: a SME perspective. Journal of cleaner production, 16(15), pp. 1579-1588 (2008)

[13] D. Dunphy. Organizational change for corporate sustainability. pp. 695-698. London: Routledge (2003)

[14] G. Keijzers. Business, Government and Sustainable Development. Oxford: Routledge (2005)

[15] Y. Agan, M.F Acar, A. Borodin. Drivers of environmental processes and their impact on performance: a study of Turkish SMEs. Journal of Cleaner Production, 51, pp 23-33 (2013)

[16] D. Morrow, D. Rondinelli. Adopting Corporate Environmental Management Systems: Motivations and Results of ISO 14001 and EMAS Certification. European Management Journal, 20(2), pp. 159-171 (2002)

[17] H.E.J. Bos-Brouwers. Corporate sustainability and innovation in SMEs: evidence of themes and activities in practice. Business Strategy and the Environment, 19(7), pp. 417-435 (2010)

[18] D.E Packham. Adhesive technology and sustainability. International Journal of Adhesion and Adhesives, 29(3), pp. 248-252 (2009) 